\title{
Preparation of Sulfur-Free Exfoliated Graphite by a Two-Step Intercalation Process and Its Application for Adsorption of Oils
}

\author{
Jun He, Laizhou Song, Hongxia Yang, Xiaohui Ren, and Lifei Xing \\ School of Environmental and Chemical Engineering, Yanshan University, Qinhuangdao 066004, China \\ Correspondence should be addressed to Laizhou Song; songlz@ysu.edu.cn
}

Received 29 March 2017; Accepted 15 May 2017; Published 6 June 2017

Academic Editor: Carola Esposito Corcione

Copyright (C) 2017 Jun He et al. This is an open access article distributed under the Creative Commons Attribution License, which permits unrestricted use, distribution, and reproduction in any medium, provided the original work is properly cited.

\begin{abstract}
The sulfur-free exfoliated graphite (EG) was prepared by a two-step chemical oxidation process, using natural flake graphite (NFG) as the precursor. The first chemical intercalation process was carried out at a temperature of $30^{\circ} \mathrm{C}$ for 50 min, with the optimum addition of NFG, potassium permanganate, and perchloric acid in a weight ratio of $1: 0.4: 10.56$. Then, in the secondary intercalation step, dipotassium phosphate was employed as the intercalating agent to further increase the exfoliated volume (EV) of EG. NFG, graphite intercalation compound (GIC), and EG were characterized by scanning electron microscope (SEM), energy dispersive spectrometer (EDS), X-ray diffractometer (XRD), Fourier transform infrared spectrometer (FTIR), BET surface area, and porosity analyzer. Also, the uptakes of crude oil, diesel oil, and gasoline by EG were determined. Results show that perchloric acid and hydrogen phosphate are validated to enter into the interlayer of graphite flake. The obtained EG possesses a large exfoliated volume (EV) and has an excellent affinity to oils; thus, the material has rapid adsorption rates and high adsorption capacities for crude oil, diesel oil, and gasoline.
\end{abstract}

\section{Introduction}

Although the petroleum consumption has resulted in a great deal of damage to natural environment [1], it is still extensively employed as one of the major fossil fuels. The increasing need of petroleum promotes the transport of this fossil; consequently, oil spill accidents have occurred and posed a serious threat to organisms and human beings [2]. Thus, for the purpose of alleviating the pollution for ocean, rivers, and lakes in the emergency accident caused by oil spill, it is urgent to dispose oil pollutants using the high efficient technology. Generally, the adsorption technique can be exactly competent for the disposal of spilled oils, so suction felt, suction linoleum, foam materials, and some porous polymers have always been employed as the adsorbents. Unfortunately, these adsorbents have an undesirable affinity to oils, thereby weakening the absorption efficiencies of them $[3,4]$. In order to alleviate the pollution caused by spilled oils, the exploitation of novel adsorbents with a high affinity to oil is very imperative. Compared with the above-mentioned adsorbents, exfoliated graphite (EG) has received tremendous attention in dealing with marine oil pollution due to its large adsorption capacity of oil and the noticeable disposal efficiency [5-8].

EG as a sort of versatile material is applied in various fields such as packing [9], sealing material [10], fire retardant [11], oil absorbing material [8], and electrode [12], due to its peculiar properties of flexibility, lubricity, and adsorption. There are three techniques applied to prepare the precursor of EG, namely, graphite intercalation compound (GIC) - gas phase intercalation, chemical oxidation, and electrochemical intercalation [13]. Among these three techniques, chemical oxidation [14] and electrochemical intercalation methods [15] are commonly used. Compared with electrochemical intercalation method, chemical oxidation method owns the merits of more convenience, lower cost, and higher stability of the products. EG with an exfoliated volume (EV) of $200-300 \mathrm{~mL} / \mathrm{g}$ can be obtained via the conventionally chemical oxidation process using concentrated sulfuric acid and concentrated nitric acid as the oxidants and intercalating reagents $[16,17]$. However, this process always releases sulfur dioxide and nitrogen oxide into the air. In this regard, the 
fabrication process of EG with the friendly environmental characteristics should be developed $[18,19]$.

The aim of this research is to develop a fabrication process of EG without using concentrated sulfuric acid and nitric acid as oxidants and intercalating reagents and then evaluate its adsorption efficiency of oils. In this study, a two-step chemical oxidation process was proposed; herein, reagents of perchloric acid $\left(\mathrm{HClO}_{4}\right)$ and dipotassium phosphate $\left(\mathrm{KH}_{2} \mathrm{PO}_{4}\right)$ were employed as intercalating agents in the first and second steps, respectively. Structures, crystallinities, components, and chemical groups of natural flake graphite (NFG), GIC, and EG were characterized by scanning electron microscopy (SEM), X-ray diffraction (XRD), energy dispersive X-ray analysis (EDS), and Fourier transform infrared spectroscopy (FTIR) techniques. Influences of intercalation temperature, reaction time, and additions of potassium permanganate $\left(\mathrm{KMnO}_{4}\right)$ and $\mathrm{HClO}_{4}$ on EV of EG were analyzed. Also, the uptakes of crude oil, diesel oil, and gasoline by EG were determined.

\section{Materials and Methods}

2.1. Materials. The NFG with an average size of $500 \mu \mathrm{m}$ was purchased from Qingdao Tianheda Graphite Co., Ltd. (Shandong, China), and the weight content of carbon is $99.5 \mathrm{wt} . \%$. Analytical reagents of $\mathrm{HClO}_{4}$ (70 72 wt.\%), $\mathrm{KMnO}_{4}$ (99.5 wt.\%), and $\mathrm{KH}_{2} \mathrm{PO}_{4}$ (99 wt.\%) were provided by Jingchun Scientific Co., Ltd. (Shanghai, China); crude oil, diesel oil, and gasoline were obtained from China National Petroleum Corporation.

2.2. Preparations of GIC and EG. GIC was prepared by a chemical oxidation process as follows. Under the continuous stirring condition, $3 \mathrm{~g}$ of $\mathrm{NFG}$ and the amount of $\mathrm{KMnO}_{4}$ $(0.3-2.1 \mathrm{~g})$ were added slowly into a glass beaker, where the amount of $\mathrm{HClO}_{4}(9-27 \mathrm{~mL})$ was previously added in this beaker. The addition of $\mathrm{KMnO}_{4}$ should be controlled discreetly to prevent the temperature of the mixture exceeding $80^{\circ} \mathrm{C}$. After the one-step intercalation reaction, $50 \mathrm{~mL}$ of saturated $\mathrm{KH}_{2} \mathrm{PO}_{4}$ solution was added to the mixture, and the oxidation process continued for $1 \mathrm{~h}$ at a temperature of $40^{\circ} \mathrm{C}$. Then, the mixed solution was filtered and the collected GIC was cleaned with distilled water until the $\mathrm{pH}$ value of the washing effluent was neutral. Lastly, the cleaned GIC was dewatered by a suction filtration process followed by drying in an oven at $65^{\circ} \mathrm{C}$ for $24 \mathrm{~h}$.

The preparation process of EG was as follows: $1 \mathrm{~g}$ of GIC was first added to a crucible, and then the crucible was placed into a muffle furnace and heated with a temperature of $950^{\circ} \mathrm{C}$ for $15 \mathrm{~s}$. During the heating treatment, the laminar structure of GIC sample was exfoliated, and EG sample was finally obtained.

2.3. Characterization. The morphologies of NFG, GIC, and EG were characterized by a Hitachi S-4800 scanning electron microscope (SEM, Hitachi, Japan); the chemical components of GIC and EG were determined by an energy dispersive spectrometer (EDS) equipped into the above-mentioned microscope. The crystalline structures of NFG, GIC, and EG were measured using a Rigaku D/max 2500 PC X-ray diffractometer (XRD, Rigaku, Japan) with $\mathrm{Cu} K \alpha$ radiation $(\lambda=1.5418 \AA)$ at $40 \mathrm{KV}$ and $30 \mathrm{~mA}$. The X-ray diffraction patterns were recorded in the range of $2 \theta=10-90^{\circ}$. After being blended with $\mathrm{KBr}$, a Nicolet IS10 Fourier transform infrared (FTIR) spectrometer (Nicolet, America) was employed to detect chemical groups of the obtained GIC and EG samples; FTIR data were recorded in the range of $4000-500 \mathrm{~cm}^{-1}$. BET surface area, pore volume, and average pore diameter of the fabricated EG were measured by mercury intrusion porosimetry using an Autopore IV 9500 analyzer (Micromeritics, USA).

The exfoliated volume (EV) was determined by exfoliating $1.0 \mathrm{~g}$ of GIC in a temperature of $950^{\circ} \mathrm{C}$ for $15 \mathrm{~s}$ and its volume was measured using a graduated cylinder. Then the volume was recorded, and this datum was considered to be EV value. In order to ensure the accuracy, measurements were carried out in triplicate, and the average value was adopted.

2.4. Adsorption Rate and Capacity of EG for Oils. Crude oil, diesel oil, and gasoline as the oil candidates were selected as the target pollutants to measure the adsorption rate and capacity of EG. Three portions of EG samples with the same weight were placed in different beakers; then the excessive target oil was added and the solution was stirred at a temperature of $25^{\circ} \mathrm{C}$. The mixture of EG and oil was separated by 35-numbered mesh net. After the different adsorption time, the collected EG sample was statically placed to make the excessive oil completely dropping down. The adsorption time was set at $1,2,4,5,6,8,10,20$, and $30 \mathrm{~min}$. The adsorption capacity in different time was obtained from the weight increase before and after adsorption. The adsorption capacity of oil at certain time was calculated via

$$
Q=\frac{M-m}{m},
$$

where $Q$ is the adsorption capacity of EG at certain time $(\mathrm{g} / \mathrm{g})$; $M$ and $m$ (g) are the weights of EG before and after the oil adsorption. In order to ensure the accuracy, measurements were carried out in triplicate, and the average value was adopted.

The regeneration ratio of $\mathrm{EG}$ (the ratio between the readsorbed oil amount and the initial adsorbed value) was assessed with $1 \mathrm{~g}$ addition of EG. EG and oil were separated through air pump filtration process; then the regenerated EG was used for oil adsorption; this process was repeated three times. The regeneration ratio is calculated by

$$
\frac{\text { regeneration ratio }(n)}{\%}=\frac{Q_{n+1}}{Q_{n}} \times 100 \%,
$$

where $n$ is the times of cyclic regeneration.

\section{Results and Discussion}

3.1. Influence of Intercalation Temperature. The effect of intercalation temperature on EV was presented in Figure 1. As shown in Figure 1, EV of EG increases with the enhancement 


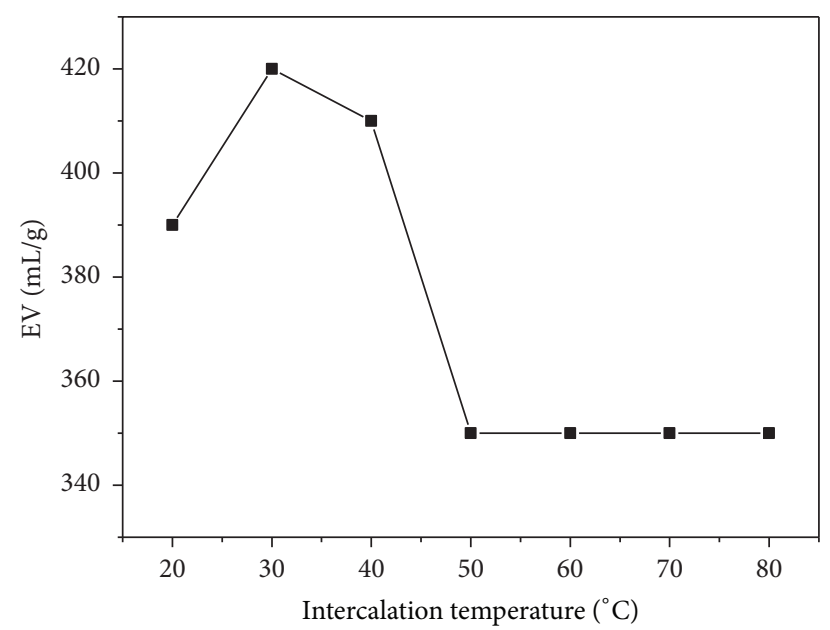

FIgURE 1: Effect of intercalation temperature on EV.

in intercalation temperature, and then it reduces when the temperature was higher than $30^{\circ} \mathrm{C}$. Thus, the temperature of $30^{\circ} \mathrm{C}$ can be identified as the optimum intercalation temperature. As the reaction time was $60 \mathrm{~min}$ and the mass ratio of NFG, $\mathrm{KMnO}_{4}$, and $\mathrm{HClO}_{4}$ was $1: 0.4: 10.56$, the value of EV reaches the maximum value $(420 \mathrm{~mL} / \mathrm{g})$.

It is well known that the preparation of GIC via the chemical oxidation technique is an exothermic process, so the low temperature can be favorable to the intercalating reaction. But a high reaction temperature (no more than $30^{\circ} \mathrm{C}$ ) might be needed at the beginning, which will be valuable for the intercalating reagent entering into the laminar spacing of NFG. However, the intercalating temperature higher than $30^{\circ} \mathrm{C}$ will accelerate the volatilization of $\mathrm{HClO}_{4}$. As a result, the amount of this agent entering the graphite interlayer reduces, thus resulting in a low EV.

3.2. Influence of Reaction Time. When the intercalating temperature and the mass ratio of NFG, $\mathrm{KMnO}_{4}$, and $\mathrm{HClO}_{4}$ were kept at $30^{\circ} \mathrm{C}$ and $1: 0.4: 10.56$, respectively, the effect of reaction time on $\mathrm{EV}$ was analyzed and the results are shown in Figure 2. The value of EV increases from 310 to $420 \mathrm{~mL} / \mathrm{g}$ as the reaction time elapsed from 10 to $50 \mathrm{~min}$, and then it slightly decreases as time extends. When the reaction time was less than $50 \mathrm{~min}$, the intercalating reaction cannot complete and the extended time will be helpful in destroying the edge layer of graphite by the oxidant. Thus, the prolonged time will guarantee more intercalating reagents entering into the laminar layer of graphite, which is beneficial to the achievement of high EV. This oxidation process is almost finished as the intercalating time is $50 \mathrm{~min}$; then EV will decrease when the time was further prolonged because intercalating reagents will run away as time goes on. Therefore, the optimum reaction time of $50 \mathrm{~min}$ was ascertained for the preparation of EG.

3.3. Influence of $\mathrm{KMnO}_{4}$ Addition. EV of EG increases significantly with the increase in $\mathrm{KMnO}_{4}$ addition (from 0.1 to $0.4 \mathrm{~g} / \mathrm{g} \mathrm{NFG}$ ) (Figure 3); then the EV change can be ignored

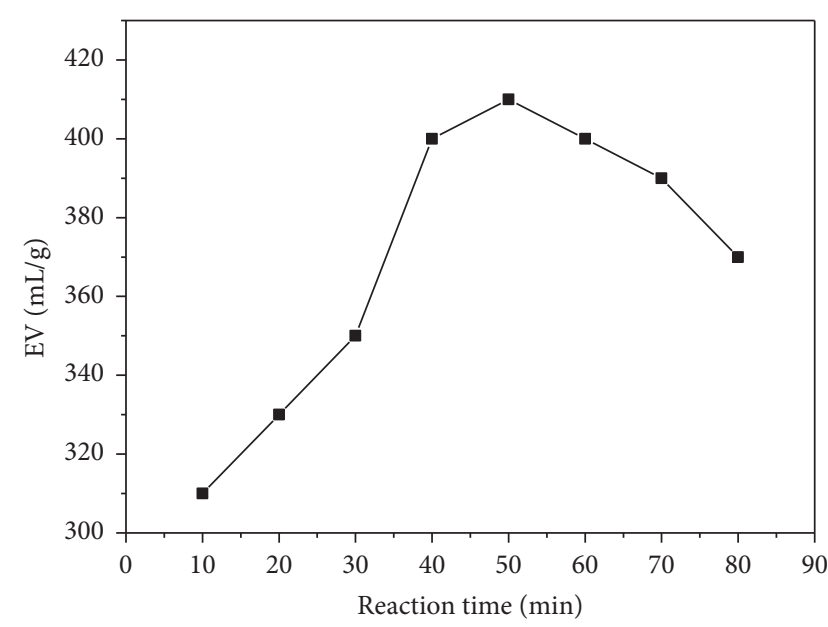

Figure 2: Effect of reaction time on EV.

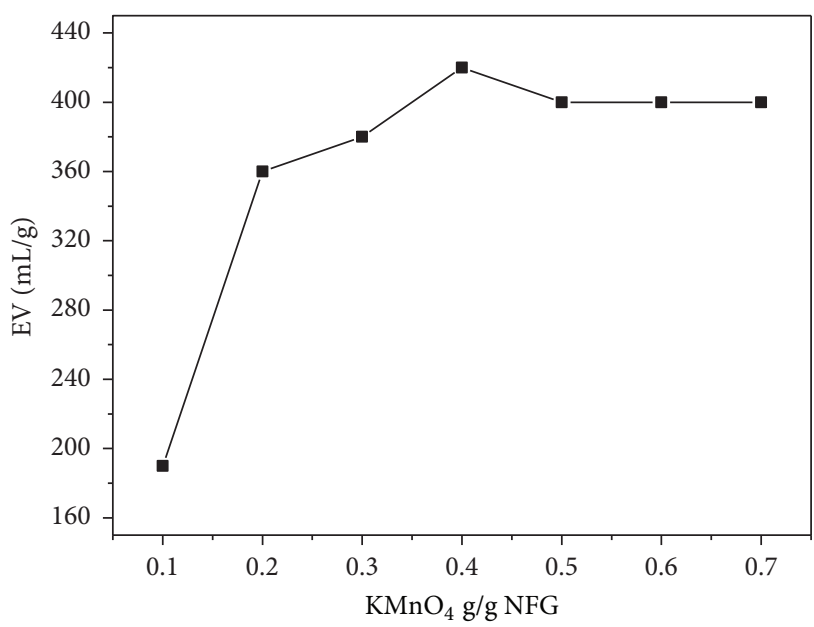

FIgURE 3: Effect of $\mathrm{KMnO}_{4}$ addition on EV.

as the addition of $\mathrm{KMnO}_{4}$ ranges from 0.5 to $0.7 \mathrm{~g} / \mathrm{g} \mathrm{NFG}$. The maximum EV with a value of $420 \mathrm{~mL} / \mathrm{g}$ can be obtained with $\mathrm{KMnO}_{4}$ addition of $0.4 \mathrm{~g} / \mathrm{g}$ NFG. During the intercalating reaction, the concentration of $\mathrm{KMnO}_{4}$ in $\mathrm{HClO}_{4}$ could hardly maintain saturated when its addition is below 0.4; as a consequence, NFG cannot be oxidized sufficiently, and the value of $\mathrm{EV}$ will be low. However, the excessive dosage of $\mathrm{KMnO}_{4}(>0.4 \mathrm{~g} / \mathrm{g} \mathrm{NFG})$ will erode the edge layers of NFG and destroy its lamellar structure. The intercalating agents will not be readily inserted into interlayer spacing, thereby resulting in the decrease in EV $(<420 \mathrm{~mL} / \mathrm{g})$. The optical addition in weight ratio of NFG and $\mathrm{KMnO}_{4}$ is validated as $1: 0.4$.

3.4. Influence of $\mathrm{HClO}_{4}$ Addition. The influence of $\mathrm{HClO}_{4}$ concentration on EV was also investigated and the result is depicted in Figure 4. Firstly, EV considerably increases with the enhancement in addition of $\mathrm{HClO}_{4}(5.28 \sim 10.56 \mathrm{~g} / \mathrm{g} \mathrm{NFG})$; but when the dosage exceeded $10.56 \mathrm{~g} / \mathrm{g}$ NFG, EV decreases slightly and stays at a stable value of $390 \mathrm{~mL} / \mathrm{g}$. At a low addition of $\mathrm{HClO}_{4}$, despite the help of $\mathrm{KMnO}_{4}$ to destroy 


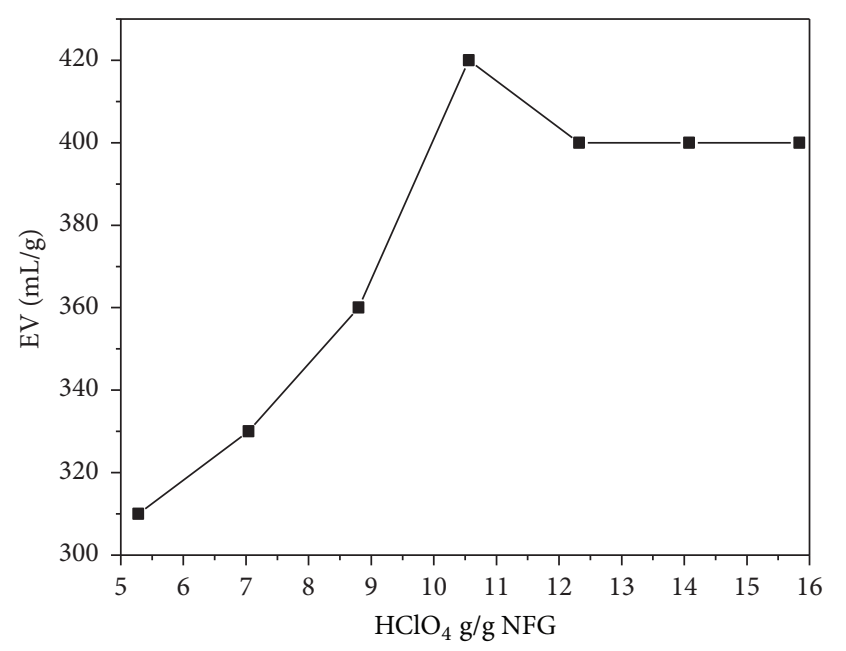

Figure 4: Effect of $\mathrm{HClO}_{4}$ addition on EV.

the lamellar structure of NFG, the amount of $\mathrm{HClO}_{4}$ was not enough to guarantee that the interlayer spacing of graphite is filled as most possible. On the contrary, at the higher addition of $\mathrm{HClO}_{4}$ (>10.56 g/g NFG), the excessive acid will dilute the concentration of $\mathrm{KMnO}_{4}$, thereby weakening the oxidation potential of this oxidant; this is unfavorable for the enhancement in EV. The optimal weight ratio between NFG and $\mathrm{HClO}_{4}$ is 1:10.56; with these additions of the above two reagents, the measured $\mathrm{EV}$ value is $420 \mathrm{~mL} / \mathrm{g}$.

In summary, the optimal conditions for the preparation of EG with EV value of $420 \mathrm{~mL} / \mathrm{g}$ are as follows: mass ratio of NFG: $\mathrm{KMnO}_{4}: \mathrm{HClO}_{4}=1: 0.4: 10.56$; the intercalating temperature $30^{\circ} \mathrm{C}$, and the reaction time $50 \mathrm{~min}$. At the aforementioned preparation condition, the EV value of the prepared EG is significantly higher than those reported in literatures [20-22].

\subsection{Characterization}

3.5.1. SEM Analysis. Morphologies of NFG, GIC, and EG under the previously described conditions were characterized, and SEM micrographs of them are presented in Figure 5. It is clear that the interlayer spacing of NFG is compact and much smaller than that of GIC (Figure 5(a)). The change in interlayer spacing between these two samples can be due to the intercalation and exfoliation of NFG. After the oxidation treatment, the interlayer spacing of NFG is enlarged and its boundary layers are crimped (Figure 5(b)). The worm-like porous structure can be seen from the surface morphology of EG (Figures 5(c)-5(f)), and the pore size ranges from several microns to hundreds of microns. The slit-shaped gaps between the graphite platelets can also be identified; this can be due to the fact that the compounds in interlayers of GIC were decomposed and some micropores are formed during the exfoliation process. The presence of the micropores will be of benefit for guaranteeing an excellent adsorption property of the prepared EG.
TABLE 1: The atomic ratios of GIC and EG.

\begin{tabular}{lcc}
\hline Atom & Atomic ratios of a & Atomic ratios of b \\
\hline $\mathrm{C}$ & 31.88 & 94.87 \\
$\mathrm{O}$ & 57.87 & 5.06 \\
$\mathrm{P}$ & 0.59 & - \\
$\mathrm{Cl}$ & 8.03 & 0.05 \\
$\mathrm{~K}$ & 0.61 & - \\
$\mathrm{Mn}$ & 1.01 & 0.02 \\
\hline
\end{tabular}

3.5.2. EDS Analysis. The chemical components of GIC and EG were determined, and measured EDS spectra are displayed in Figure 6. The chloride $(\mathrm{Cl})$ and phosphorus $(\mathrm{P})$ elements can be identified on the surfaces of tested samples. For both GIC and EG, the determined elements (atomic weight) are shown in Table 1. Atomic weights of $\mathrm{Cl}$ and $\mathrm{P}$ in GIC are $8.03 \%$ and $0.95 \%$, respectively. The existence of $\mathrm{Cl}$ and $\mathrm{P}$ may derive from the additions of $\mathrm{HClO}_{4}$ and $\mathrm{KH}_{2} \mathrm{PO}_{4}$. Thus, it can be deduced that both $\mathrm{HClO}_{4}$ and $\mathrm{KH}_{2} \mathrm{PO}_{4}$ were inserted into the interlayer of NFG; this process is a prerequisite for the preparation of EG. But the content of $\mathrm{Cl}$ in $\mathrm{EG}$ decreases to $0.05 \%$ and $\mathrm{P}$ cannot be detected. After the exfoliation treatment at $950^{\circ} \mathrm{C}$, the inserted agents were decomposed and the worm-like EG was obtained.

3.5.3. XRD Analysis. The tested XRD patterns of NFG, GIC, and EG are shown in Figure 7. The peak appearing at $2 \theta=$ $26.42^{\circ}$ (Figure $7(\mathrm{a})$ ) with a $d$-spacing of $3.37 \AA$ for NFG is observed. For the sample of GIC, this peak with a $d$-spacing of $3.50 \AA$ shifts to $25.40^{\circ}$ (Figure 7(b)). This can be due to the fact that $\mathrm{KMnO}_{4}$ can easily oxidize the graphite layers, and the repulsion interactions between the layers increase, thereby resulting in the increase in the interlayer spacing. As a result, $\mathrm{HClO}_{4}$ migrates into the graphite layers and expands the lamellar spacing along the $c$-axis direction, which leads to the peak intensity of GIC lower than that of NFG. The similarity of these two peaks mentioned above evidences the insufficient oxidation reaction. As for the XRD pattern of GIC, another peak at $2 \theta=30.02^{\circ}$ (Figure 7(b)) can be observed, suggesting that NFG is successfully intercalated by $\mathrm{HClO}_{4}$. Also, for the XRD pattern of GIC, it is easy to find that the reflection peak at $2 \theta=54.56^{\circ}$ is divided into two peaks $\left(2 \theta=51.88^{\circ}\right.$ and $56.94^{\circ}$ ). This change can also be attributed to the oxidation reaction. Two diffraction peaks $\left(2 \theta=26.44^{\circ}\right.$ and $\left.54.54^{\circ}\right)$ for EG are observed in Figure 7(c), but no significant difference in their locations can be identified between EG and NFG [21]. The crystallinity of EG is still analogous to that of NFG; between them, however, the discernable difference in diffraction intensities of the above peaks can be attested. After the exfoliation treatment at $950^{\circ} \mathrm{C}$, the inserted agents will be decomposed to gaseous compounds and they escape from the edges of the particles, leaving spaces between the layers. Thus, the crystal structure of EG was partially destroyed, so the decrease in crystallinity for EG samples is validated [23].

3.5.4. Analysis of FTIR Spectra. The determined FTIR spectra of GIC and EG are shown in Figure 8. The peaks at 3450 
TABLE 2: BET results of EG.

\begin{tabular}{lccc}
\hline Sample & $\begin{array}{c}\text { BET surface area } \\
\left(\mathrm{m}^{2} / \mathrm{g}\right)\end{array}$ & $\begin{array}{c}\text { Total pore volume } \\
(\mathrm{mL} / \mathrm{g})\end{array}$ & $\begin{array}{c}\text { Average pore diameter } \\
(\mu \mathrm{m})\end{array}$ \\
\hline NFG & 0.7 & - & - \\
GIC & 0.8 & - & - \\
EG & 245 & 37.5 & 22.1 \\
\hline
\end{tabular}

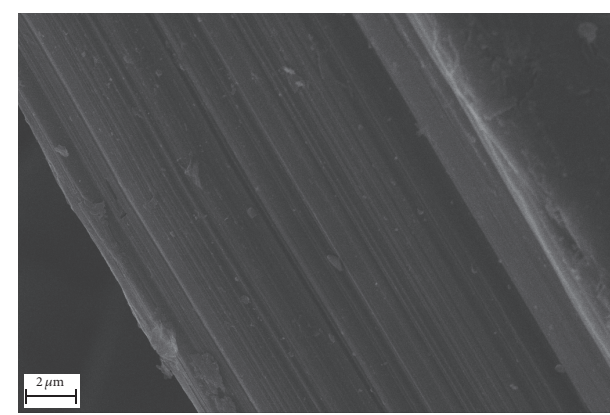

(a)

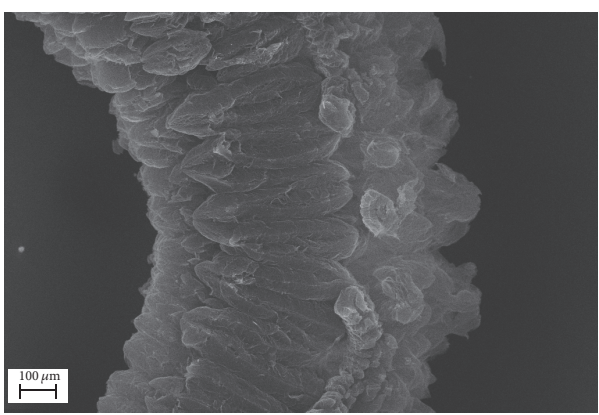

(c)

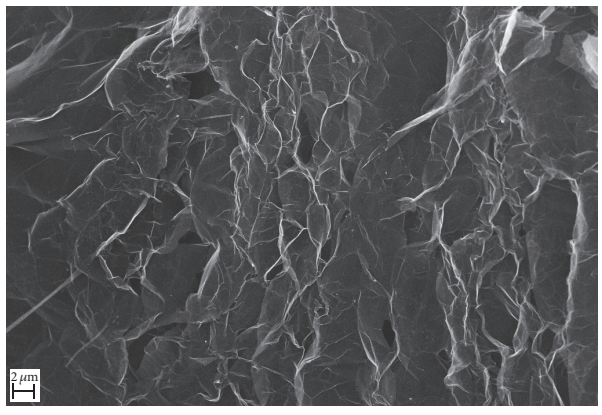

(e)

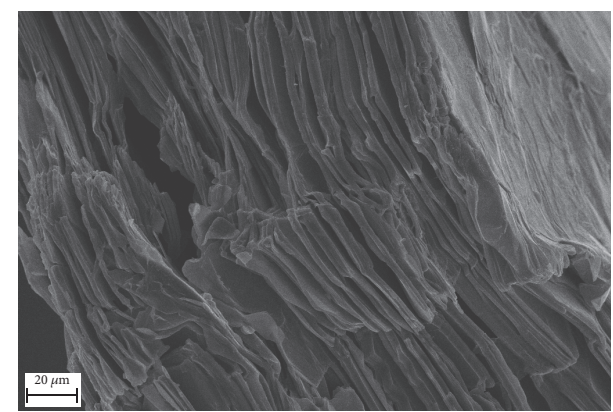

(b)

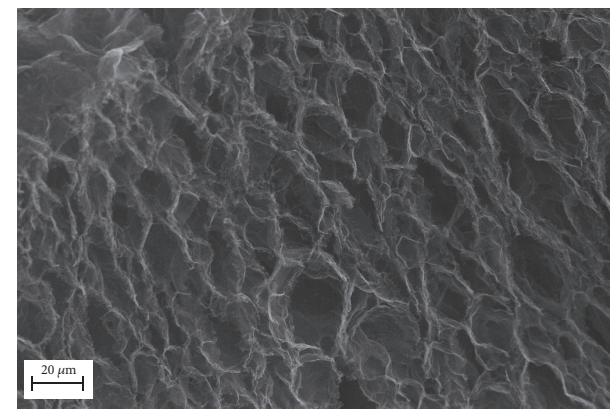

(d)

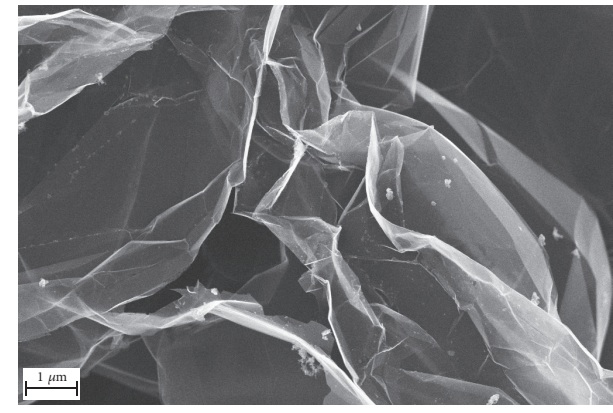

(f)

FIGURE 5: SEM images of (a) sectional morphology of natural flake graphite, (b) sectional morphology of GIC, and (c-f) surface morphology of EG: the magnification of each image is listed as follows: (a) $\times 5000$, (b) $\times 500$, (c) $\times 100$, (d) $\times 500,(e) \times 2000$, and (f) $\times 10000$.

and $1630 \mathrm{~cm}^{-1}$ (for GIC) and those at 3444 and $1624^{-1} \mathrm{~cm}$ (for EG) are derived from adsorbed water [22]. The peak of GIC at $1397 \mathrm{~cm}^{-1}$ is ascribed to hydroxyl groups because of the oxidation of NFG [24]. The presence of two peaks of GIC $\left(625\right.$ and $\left.1112 \mathrm{~cm}^{-1}\right)$ suggests the existence of $\mathrm{ClO}_{4}{ }^{-}$, and other peaks (1121-1136 $\mathrm{cm}^{-1}$ ) can be assigned to $\mathrm{H}_{2} \mathrm{PO}_{4}{ }^{-}$. Therefore, reagents of $\mathrm{HClO}_{4}$ and $\mathrm{KH}_{2} \mathrm{PO}_{4}$ are further ascertained to enter into graphite layers, being helpful in the preparation of EG.
3.5.5. Analysis of BET and Pore Structure. The surface area of NFG and GIC is low because they are bulk materials with an average size of $500 \mu \mathrm{m}$ and less of porosity. BET surface area, pore volume, and pore diameter for EG sample were measured, and results are summarized in Table 2 . The results show the feature of EG sample, that is, high surface area and micron pore structure. The surface area of the prepared EG was calculated to be $245 \mathrm{~m}^{2} / \mathrm{g}$, which is much higher than that of exfoliated graphite synthesized by microwave irradiation [8]. It is 


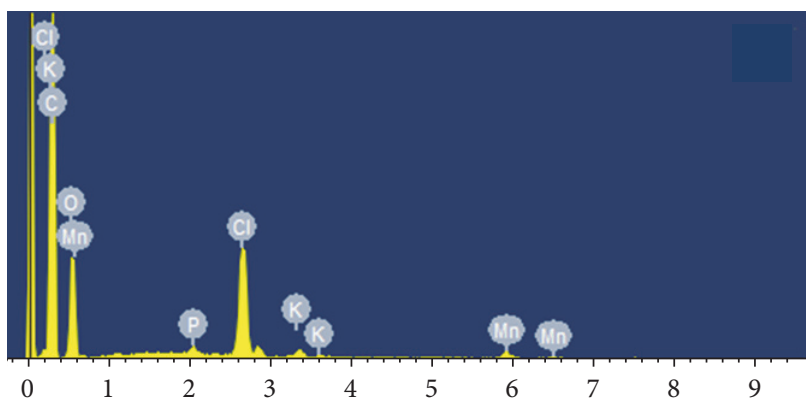

(a)

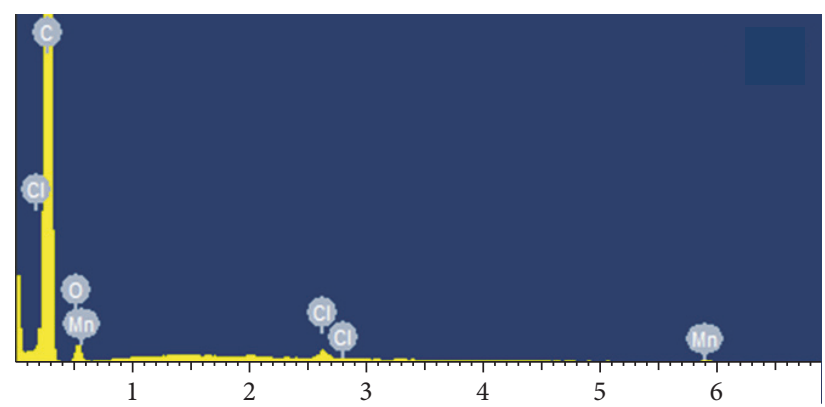

(b)

FIGURE 6: EDS spectra of (a) GIC and (b) EG.

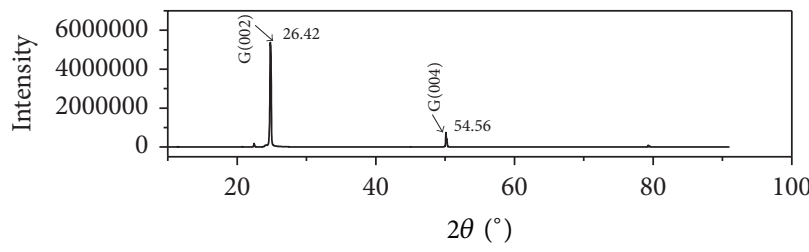

(a)

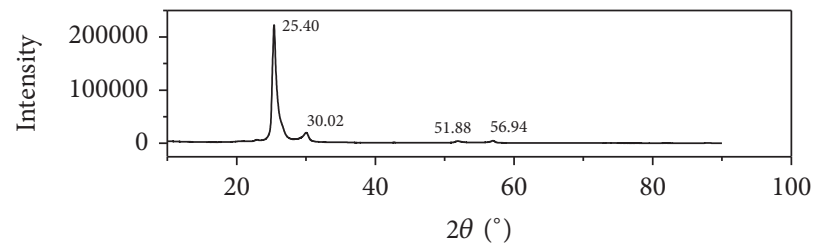

(b)

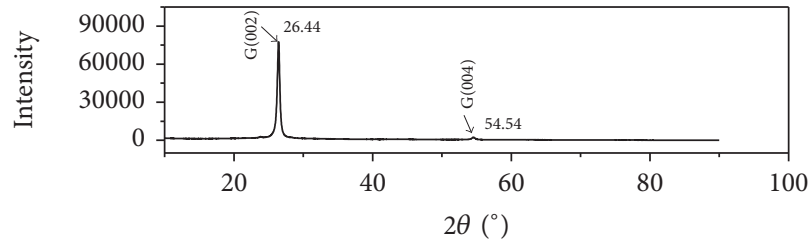

(c)

FIGURE 7: XRD patterns of (a) NFG, (b) GIC, and (c) EG.

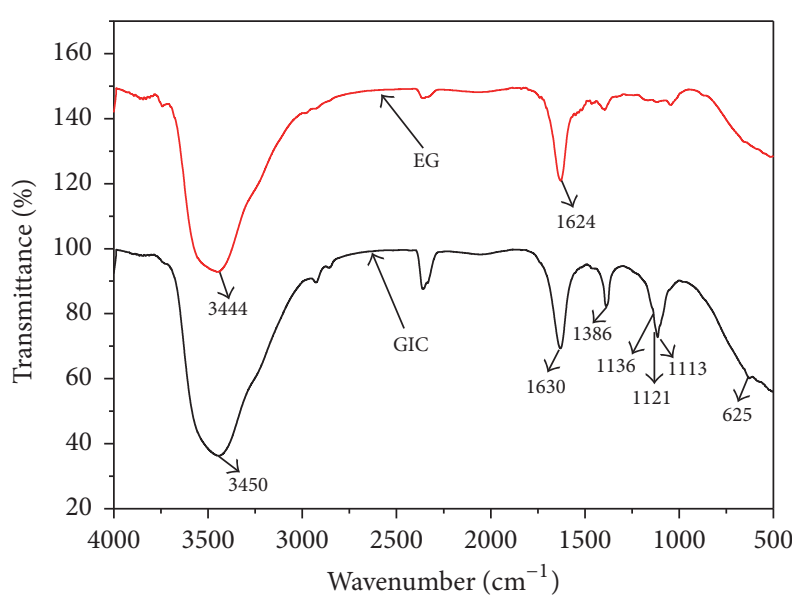

FIgURE 8: FTIR spectra of GIC and EG.

worth mentioning that the micrometer level of average pore diameter of EG will be advantageous to significantly enhance the adsorption performance toward oils $[25,26]$.

3.6. Adsorption Capacity and Adsorption Rate of EG for Crude Oil, Diesel Oil, and Gasoline. Figure 9 displays dynamic adsorption behaviors of EG for crude oil, diesel oil, and gasoline. The maximum adsorption capacities are $123.3 \mathrm{~g} / \mathrm{g}$ for crude oil, $76.5 \mathrm{~g} / \mathrm{g}$ for diesel oil, and $61.4 \mathrm{~g} / \mathrm{g}$ for gasoline. The adsorption rate is another important factor during adsorption process. The time to reach the equilibrium sorption capacity for each oil is 5, 2, and $2 \mathrm{~min}$, respectively, reflecting rapid adsorption rates for these oils. These results indicate that the as-prepared EG will be competent for the application in oil spill accidents. Herein, the adsorption characteristics of EG toward the oil will be comprehensively investigated in the further research.

3.7. Cyclic Regeneration of EG. Table 3 exhibits cyclic regeneration abilities of EG after adsorbing the aforesaid three oils. The adsorption capacity of EG for each oil decreases slightly after regeneration, confirming that EG can be repeatedly applied as an effective adsorbent after the separation process by air pump filtration.

3.8. Mechanism of Adsorption. In the process of GIC sample preparation, $\mathrm{HClO}_{4}$ is used as both an intercalation agent and oxidizing agent. The strong acidity, volatility, and oxidability of $\mathrm{HClO}_{4}$ can lead to a high-temperature expansion and the formation of microns pores for EG sample, guaranteeing its 
TABLE 3: Cyclic regeneration of EG for the three kinds of oils.

\begin{tabular}{lcccccc}
\hline Categories of oils & $\begin{array}{c}\text { Adsorption } \\
\text { capacity number } \\
\text { 1/g }\end{array}$ & $\begin{array}{c}\text { Adsorption } \\
\text { capacity number } \\
2 / \mathrm{g}\end{array}$ & $\begin{array}{c}\text { Adsorption } \\
\text { capacity number } \\
3 / \mathrm{g}\end{array}$ & $\begin{array}{c}\text { Regeneration } \\
\text { ratio (1)/\% }\end{array}$ & $\begin{array}{c}\text { Regeneration } \\
\text { ratio (2)/\% }\end{array}$ \\
$\begin{array}{l}\text { regeneration } \\
\text { ratio/\% }\end{array}$ \\
\hline Crude oil & 45.6 & 40.3 & 34.5 & 88.38 & 85.61 & 86.99 \\
Diesel oil & 27.8 & 23.6 & 19.4 & 84.89 & 82.20 & 83.29 \\
Gasoline & 23.5 & 19.8 & 15.6 & 84.25 & 78.79 \\
\hline
\end{tabular}

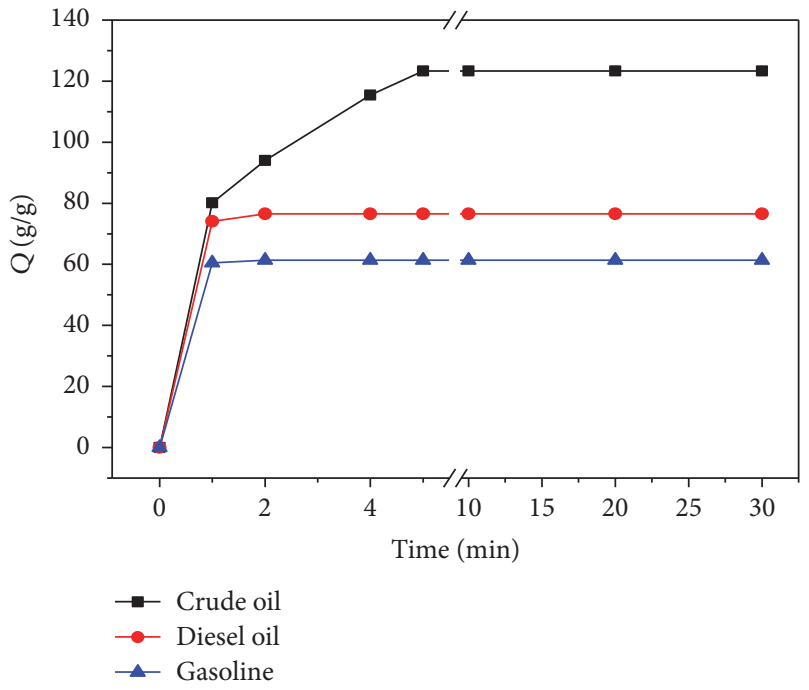

FIGURE 9: Dynamic adsorption curves of EG for crude oil, diesel oil, and gasoline.

excellent adsorption properties toward oils. In addition, high BET surface area and large pore volume of EG will also ensure rapid adsorption rates and high adsorption capacities.

\section{Conclusions}

In this study, a facile two-step intercalation method is proposed to prepare the sulfur-free EG, using $\mathrm{HClO}_{4}$ and $\mathrm{KH}_{2} \mathrm{PO}_{4}$ as the inserting agent and $\mathrm{KMnO}_{4}$ employed as the oxidant. The oxidant can easily open the graphite layers and the inserting agent will move into the interlayer spacing to obtain the GIC sample. The optimum conditions for the preparation of GIC are as follows: the mass ratio of NFG, $\mathrm{KMnO}_{4}$, and $\mathrm{HClO}_{4}$ is $1: 0.4: 10.56$; the reaction temperature and time are kept at $30^{\circ} \mathrm{C}$ and $50 \mathrm{~min}$. EV of the prepared EG is $420 \mathrm{~mL} / \mathrm{g}$. The worm-like porous morphology, high BET surface area, and large pore volume of EG guarantee the excellent adsorption property toward oils. The maximum adsorption capacities of EG for crude oil, diesel oil, and gasoline are $123.3,76.5$, and $61.4 \mathrm{~g} / \mathrm{g}$, respectively.

\section{Conflicts of Interest}

The authors declare that there are no conflicts of interest regarding the publication of this paper.

\section{Acknowledgments}

This research was funded by Hebei Provincial Science and Technology Support Program of China (Grant no. 15273613).

\section{References}

[1] M. Toyoda and M. Inagaki, "Sorption and recovery of heavy oils by using exfoliated graphite," Spill Science and Technology Bulletin, vol. 8, no. 5-6, pp. 467-474, 2003.

[2] G. Wang, Q. Sun, Y. Zhang, J. Fan, and L. Ma, "Sorption and regeneration of magnetic exfoliated graphite as a new sorbent for oil pollution,” Desalination, vol. 263, no. 1-3, pp. 183-188, 2010.

[3] A. Li, R. Lin, C. Lin et al., "An environment-friendly and multifunctional absorbent from chitosan for organic pollutants and heavy metal ion," Carbohydrate Polymers, vol. 148, pp. 272-280, 2016.

[4] S. O. Alayande, E. O. Dare, F. O. G. Olorundare, D. Nkosi, T. A. M. Msagati, and B. B. Mamba, "Superoleophillic electrospun polystrene/exofoliated graphite fibre for selective removal of crude oil from water," Physics and Chemistry of the Earth, vol. 92, pp. 3-6, 2016.

[5] C. Liu, Z. Chen, X. Cheng, Z. Wang, and X. Duan, "Preparation and structure analysis of expanded graphite-based composites made by phosphoric acid activation," Journal of Porous Materials, vol. 17, no. 4, pp. 425-428, 2010.

[6] V. G. Makotchenko, E. D. Grayfer, A. S. Nazarov, S.-J. Kim, and V. E. Fedorov, "The synthesis and properties of highly exfoliated graphites from fluorinated graphite intercalation compounds," Carbon, vol. 49, no. 10, pp. 3233-3241, 2011.

[7] B. Tryba, J. Przepiórski, and A. W. Morawski, "Influence of chemically prepared $\mathrm{H}_{2} \mathrm{SO}_{4}$-graphite intercalation compound (GIC) precursor on parameters of exfoliated graphite (EG) for oil sorption from water," Carbon, vol. 41, no. 10, pp. 2013-2016, 2003.

[8] N. Sykam and K. K. Kar, "Rapid synthesis of exfoliated graphite by microwave irradiation and oil sorption studies," Materials Letters, vol. 117, no. 15, pp. 150-152, 2014.

[9] B. Debelak and K. Lafdi, "Use of exfoliated graphite filler to enhance polymer physical properties," Carbon, vol. 45, no. 9, pp. 1727-1734, 2007.

[10] N. E. Sorokina, A. V. Redchitz, S. G. Ionov, and V. V. Avdeev, "Different exfoliated graphite as a base of sealing materials," Journal of Physics and Chemistry of Solids, vol. 67, no. 5-6, pp. 1202-1204, 2006.

[11] B. Dittrich, K.-A. Wartig, D. Hofmann, R. Mülhaupt, and B. Schartel, "Flame retardancy through carbon nanomaterials: carbon black, multiwall nanotubes, expanded graphite, multi-layer 
graphene and graphene in polypropylene," Polymer Degradation and Stability, vol. 98, no. 8, pp. 1495-1505, 2013.

[12] M. Kujawski, J. D. Pearse, and E. Smela, "Elastomers filled with exfoliated graphite as compliant electrodes," Carbon, vol. 48, no. 9, pp. 2409-2417, 2010.

[13] X. Van Heerden and H. Badenhorst, "The influence of three different intercalation techniques on the microstructure of exfoliated graphite," Carbon, vol. 88, article 9752, pp. 173-184, 2015.

[14] Y. Chen, R. Luo, S. Li et al., "Preparation of highly-expandable graphite using waste liquid propellants of nitric-27S as one of intercalating agents," Carbon, vol. 50, no. 5, p. 2063, 2012.

[15] H. M. A. Asghar, S. N. Hussain, H. Sattar, N. W. Brown, and E. P. L. Roberts, "Environmentally friendly preparation of exfoliated graphite," Journal of Industrial and Engineering Chemistry, vol. 20, no. 4, pp. 1936-1941, 2014.

[16] M. Inagaki, R. Tashiro, Y.-I. Washino, and M. Toyoda, "Exfoliation process of graphite via intercalation compounds with sulfuric acid," Journal of Physics and Chemistry of Solids, vol. 65, no. 2-3, pp. 133-137, 2004.

[17] R. Bissessur and S. F. Scully, "Intercalation of solid polymer electrolytes into graphite oxide," Solid State Ionics, vol. 178, no. 11-12, pp. 877-882, 2007.

[18] Jihui-Li, Huifang-Da, Qian-Liu, and Shufen-Liu, "Preparation of sulfur-free expanded graphite with $320 \mu \mathrm{m}$ mesh of flake graphite," Materials Letters, vol. 60, no. 29-30, pp. 3927-3930, 2006.

[19] J. Li, J. Li, and M. Li, "Ultrasound irradiation prepare sulfur-free and lower exfoliate-temperature expandable graphite," Materials Letters, vol. 62, no. 14, pp. 2047-2049, 2008.

[20] Y.-P. Chen, S.-Y. Li, R.-Y. Luo, X.-M. Lu, and X.-J. Wang, "Optimization of initial redox potential in the preparation of expandable graphite by chemical oxidation," New Carbon Materials, vol. 28, no. 6, pp. 435-441, 2013.

[21] X. H. Wei, L. Liu, J. X. Zhang, J. L. Shi, and Q. G. Guo, "HClO4graphite intercalation compound and its thermally exfoliated graphite," Materials Letters, vol. 63, no. 18-19, pp. 1618-1620, 2009.

[22] Z. Ying, X. Lin, Y. Qi, and J. Luo, "Preparation and characterization of low-temperature expandable graphite," Materials Research Bulletin, vol. 43, no. 10, pp. 2677-2686, 2008.

[23] K.-C. Tsai, H.-C. Kuan, H.-W. Chou, C.-F. Kuan, C.-H. Chen, and C.-L. Chiang, "Preparation of expandable graphite using a hydrothermal method and flame-retardant properties of its halogen-free flame-retardant HDPE composites," Journal of Polymer Research, vol. 18, no. 4, pp. 483-488, 2011.

[24] S. Stankovich, R. D. Piner, S. T. Nguyen, and R. S. Ruoff, "Synthesis and exfoliation of isocyanate-treated graphene oxide nanoplatelets," Carbon, vol. 44, no. 15, pp. 3342-3347, 2006.

[25] S. J. Yang, J. H. Kang, H. Jung, T. Kim, and C. R. Park, "Preparation of a freestanding, macroporous reduced graphene oxide film as an efficient and recyclable sorbent for oils and organic solvents," Journal of Materials Chemistry A, vol. 1, no. 33, pp. 9427-9432, 2013.

[26] S. Kabiri, D. N. H. Tran, T. Altalhi, and D. Losic, "Outstanding adsorption performance of graphene-carbon nanotube aerogels for continuous oil removal," Carbon, vol. 80, no. 1, pp. 523533, 2014. 

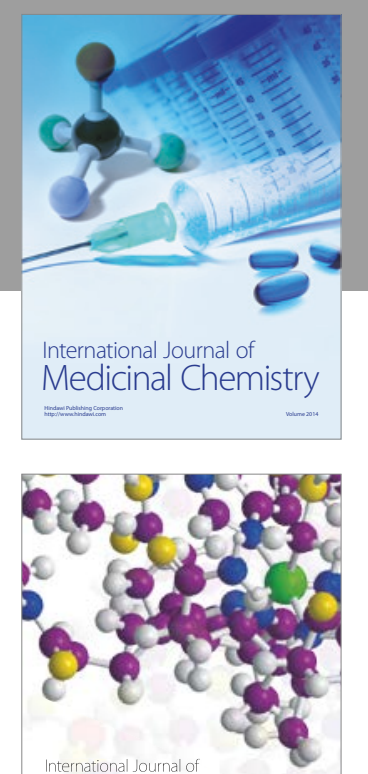

Carbohydrate Chemistry

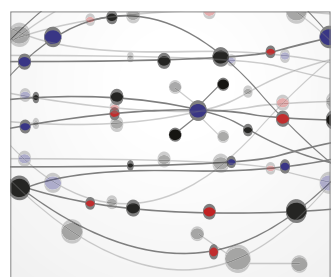

The Scientific World Journal
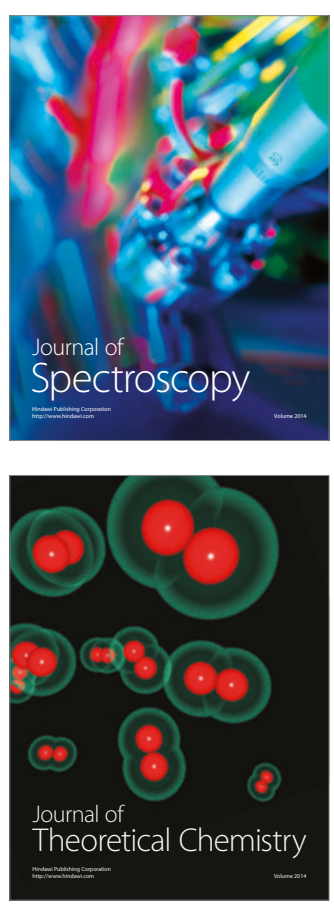
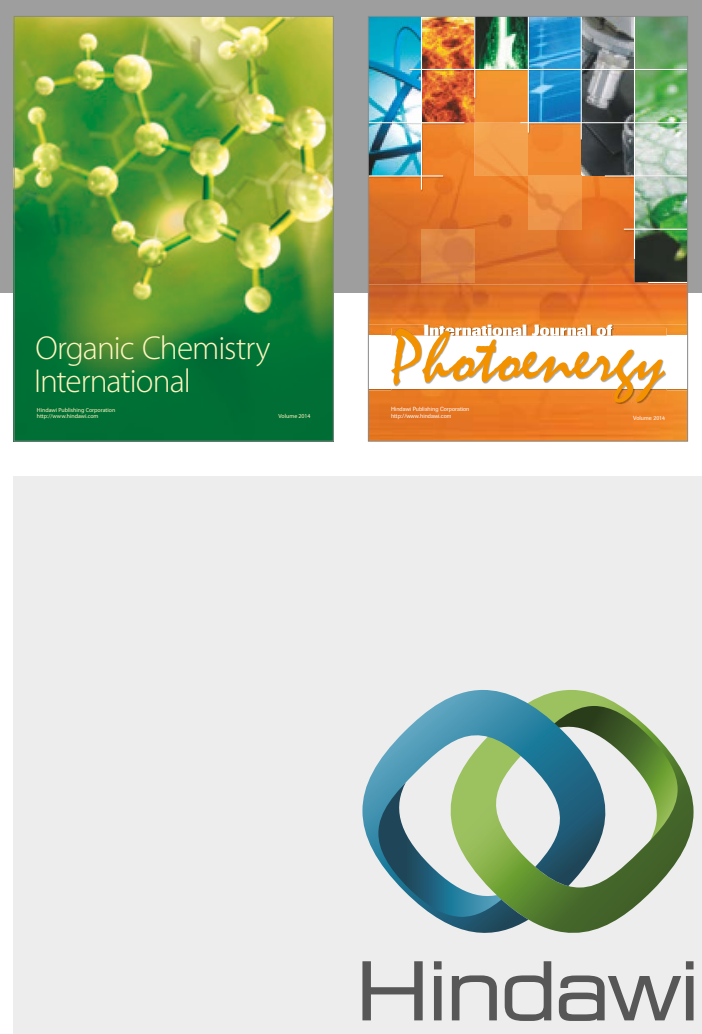

Submit your manuscripts at

https://www.hindawi.com

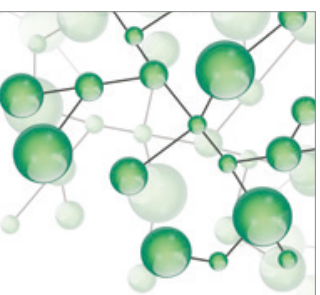

International Journal of

Inorganic Chemistry

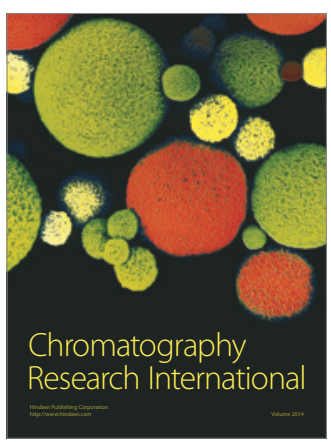

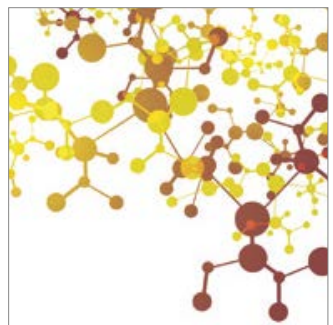

Applied Chemistry
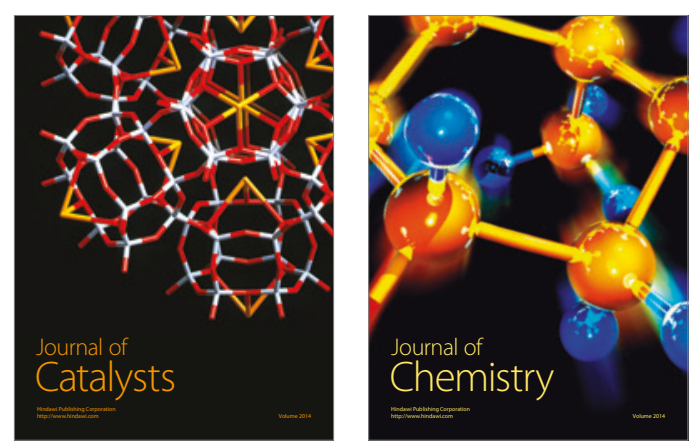
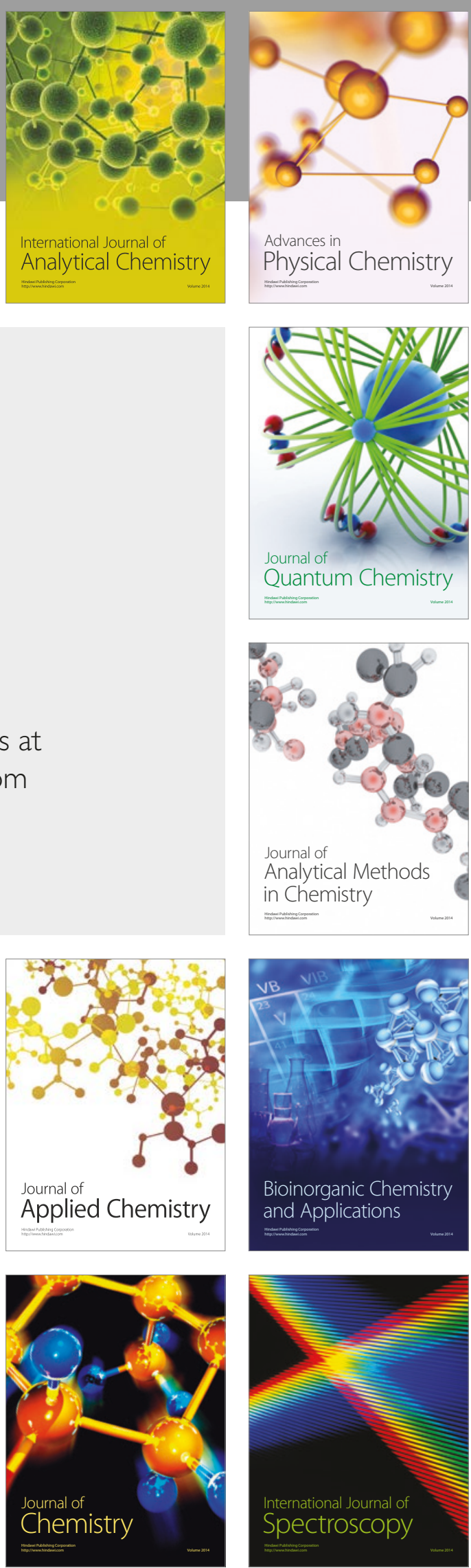